\title{
De Novo Synthesis of PCR Templates for the Development of SARS Diagnostic Assay
}

\author{
Jia Zhang, ${ }^{1}$ Bo Meng, ${ }^{2}$ Duanfang Liao, ${ }^{3}$ Lvyi Zhou, ${ }^{2}$ Xu Zhang, ${ }^{1}$ Linling Chen, ${ }^{1}$ \\ Zifen Guo, ${ }^{1}$ Cuiying Peng, ${ }^{1}$ Bingyang Zhu, ${ }^{3}$ Peggy P.Lee, ${ }^{4}$ Xiangmin $X u^{5}$ \\ Tianhong Zhou, ${ }^{6}$ Zemin Deng, ${ }^{1,7}$ Yinghe Hu, ${ }^{2}$ and Kai $\mathrm{Li}^{1,3,6,{ }^{*}}$
}

\begin{abstract}
A novel coronavirus was identified as the cause for severe acute respiratory syndrome (SARS). The complete sequence of SARS genome has provided an opportunity for the development of molecular diagnostic assays. To restrain further outbreak of SARS, the World Health Organization has posted several pairs of polymerase chain reaction (PCR) primers for early diagnosis and urged more research to be done on PCR protocols. Here we report a strategy for the de novo synthesis of PCR templates complimentary to the SARS virus genome, which has the advantage of working on PCR templates without concern about viral infection and also has the advantage that it can be used by those who do not have access to the SARS virus. This highly efficient and safe strategy for obtaining SARS gene fragments is useful for the development of PCR assays, as well as for the preparation of reliable positive controls for PCR testing kits.
\end{abstract}

Index Entries: Gene synthesis; SARS; PCR, sequential primer extension.

\section{Introduction}

Since the initial completion of the SARS genome sequenced by a Canadian group on April 13, 2003 (1), a few polymerase chain reaction (PCR) protocols have been developed from several independent laboratories (2-4). However, a clinically oriented PCR diagnostic assay with high efficiency and reliability is not yet available. Although the worldwide outbreak of SARS has caused the loss of about one thousand lives (5), most research laboratories that have molecular or genetic expertise in China, and perhaps in other areas of the world, do not currently have access to the SARS virus owing to official regulation. The potential risk of an outbreak from experimental failure also limited the effort in developing a diagnostic assay for SARS.

In the present study, we describe a new strategy for de novo synthesis of deoxyribonucleic acid (DNA) fragments complimentary to the SARS genome. By using a set of partially overlapping oligonucleotides, specific templates were quickly obtained by sequential primer extension. This strategy is simple and safe with no requirement for sophisticated equipment and does not need a highly regulated experimental environment. In addition to its urgent application in the development of the SARS diagnostic assay, this new strategy can also be used in the synthesis of genes, in vitro, for expression studies, especially for mutagenesis studies.

\section{Material and Methods 2.1. Design of Overlapped Primer Set}

Two sets of primers, set A and set B, synthesized by Sangon (Shanghai, China) were used in the sequential primer extension. Each of the sets contained three forward primers and one reverse

\footnotetext{
*Author to whom all correspondence and reprint requests should be addressed: ${ }^{1}$ Genomapping Inc., Tianjin. ${ }^{2}$ Shanghai Institute of Brain Functional Genomics, Esat China Normal University, Shanghai. ${ }^{3}$ Institute of SNP, Institute of Pharmacy and Pharmacology, Nanhua University, Hengyang. ${ }^{4}$ University of California in San Diego, San Diego, USA. ${ }^{5}$ Department of Medical Genetics, The First Military Medical University, Guangzhou. ${ }^{6}$ Institute of Life Science and Biotechnology, Jinana University, Guangzhou. ${ }^{7}$ Genomics Group, Loma Linda University, CA. *kaili34@yahoo.com. Tel.: (858)549-8830.
}

Molecular Biotechnology @ 2003 Humana Press Inc. All rights of any nature whatsoever reserved. 1073-6085/2003/25:2/107-112/\$25.00 
Table 1

Sequences of Primers Used in Sequential Primer Extension

\begin{tabular}{|c|c|}
\hline$\underline{\text { Primers }}$ & Sequence \\
\hline \multicolumn{2}{|l|}{ Set A } \\
\hline AF1 & tgataagagctacgagcaccagacacccttcgaaattaagagtgccaagaaatttgacactttcaaaggggaatgcc \\
\hline AF2 & ctttcaaaggggaatgcccaaagtttgtgtttcctcttaactcaaaagtcaaagtcattcaaccacg \\
\hline AF3 & caaagtcattcaaccacgtgttgaaaagaaaaagactgagggtttcatggggcgtatacgctctgtgt \\
\hline AR & 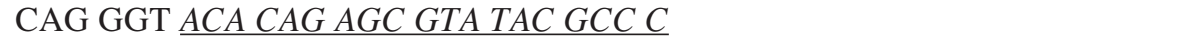 \\
\hline \multicolumn{2}{|l|}{ Set B } \\
\hline BF1 & attgtctcctggtttactggctacaaacaatgtctttcgcttaaaagggggtgcaccaattaaaggtgtaacctttggag \\
\hline BF2 & aaggtgtaacctttggagaagatactgtttgggaagttcaaggttacaagaatgtgagaatcacatt tgagcttgat \\
\hline BF3 & atcacatttgagcttgatgaacgtgttgacaaagtgcttaatgaaaagtgctctgtctacactgttgaatccggtac \\
\hline BR & ACT TCG $\underline{G T A} C C G$ GAT TCA ACA GTG T \\
\hline
\end{tabular}

Note: $\mathrm{AR}$ and $\mathrm{BR}$ are reverse primers that were the starting points for the sequential extension. The underlined bases represent the portions of the sequences that were overlapped.

primer. All primers within one set were partially overlapped (Table 1). The first forward primer and the reverse primer of each set were chosen after being blasted within the Genbank to assure that these were conserved regions among the SARS strains so far identified and to avoid potential nonspecific products.

\subsection{Sequential Primer Extension}

Primer extension conditions are described elsewhere in detail (6). Briefly, primer extension was carried out for 30 cycles with $30 \mathrm{~s}$ denaturation at $95^{\circ} \mathrm{C}, 1 \mathrm{~min}$ annealing at $56^{\circ} \mathrm{C}$, and 1 min extension at $72^{\circ} \mathrm{C}$. The primer extension reaction was performed in a total volume of $25 \mu \mathrm{L}$ with no template, $0.2 \mathrm{~m} M$ deoxynucleotide 5'-triphosphate (dNTP), $10 \mathrm{pmol} / \mathrm{mL}$ of both sense and antisense primers, $2.5 \mu \mathrm{L}$ of the $10 \mathrm{X}$ NEB polymerase reaction buffer (New England Biolab, Beverly, MA), and 0.625 U Taq polymerases (Takara, Dalian, Liaoling). PCR products were visualized using $2 \%$ agarose gel electrophoresis running under $10 \mathrm{~V} / \mathrm{cm}$. Two types of sequential primer extension were performed: single-step sequential primer extension and consecutive-step sequential primer extension.

\subsubsection{Protocol 1. Single-Step Sequential Primer Extension}

All four primers in primer set A, designed for the production of Temp-I, or primer set $\mathrm{B}$, de- signed for Temp-II, were added to one reaction system and a different concentration for each primer was used in this sequential primer extension assay. The final concentration of the first forward primers of $\mathrm{AF} 1$ and $\mathrm{BF} 1$ and the reverse primers of $\mathrm{AR}$ and $\mathrm{BR}$ was $10 \mu \mathrm{M}$; the concentration of the second forward primers of AF2 and BF2 was $0.5 \mu M$, and the concentration of the third forward primers of AF3 and BF3 was 0.025 $\mu M$. This single-step sequential primer extension was expected to yield mainly final templates with no intermediate products.

\subsubsection{Protocol 2. Consecutive-Step Sequential Primer Extension}

The number of separate reactions in consecutive-step sequential primer extension is as many as the number of forward primers. Step 1 uses a reverse primer together with the most 3 ' of the forward primers. Thereafter, the product of each reaction serves as the reverse primer for the next step. A method similar to this was described by Horton et al. (7). In this manner, the doublestranded products propagate from reverse primer toward the forward primers bridged by the overlapped sequences. As shown in Table 2, in the initial steps, forward primers AF3 or BF3 and the reverse primers AR or BR were the only oligonucleotides within the reaction system and were used at a final concentration of $10 \mu M$. For all the 
Table 2

Corresponding Products From Each Step of the Sequential Primer Extension

\begin{tabular}{|c|c|c|c|}
\hline $\begin{array}{l}\text { Sequential } \\
\text { primer } \\
\text { extension }\end{array}$ & $\begin{array}{c}\text { Double } \\
\text { strand converted }\end{array}$ & Expected products $^{a}$ & $\begin{array}{l}\text { Product size } \\
\text { (bp) }\end{array}$ \\
\hline \multicolumn{4}{|l|}{ Set A } \\
\hline Step 1 & AF3_AR & ...tcatg gggcgtatacgctctgt gtaccctg $3^{\prime}$ & 74 \\
\hline Step 2 & AF2_AF3-AR & ...aaaag tcaaagtcattcaaccac gtgtt & 123 \\
\hline Step 3 & $\mathrm{AF} 1 \_\mathrm{AF} 2-\mathrm{AF} 3-\mathrm{AR}$ & ...5' tgaca ctttcaaaggggaatgcc caaag & 182 \\
\hline \multicolumn{4}{|l|}{ Set B } \\
\hline Step 1 & BF3_BR & ...tgtct acactgttgaatccggtaccgaagt $3^{\prime}$ & 83 \\
\hline Step 2 & $\mathrm{BF} 2 \_\mathrm{BF} 3-\mathrm{BR}$ & ...tgaga atcacatttgagcttgat gaacg & 142 \\
\hline Step 3 & $\mathrm{BF} 1 \_\mathrm{BF} 2-\mathrm{BF} 3-\mathrm{BR}$ & ...5' aatta aaggtgtaacctttggagg aagat & 204 \\
\hline
\end{tabular}

${ }^{a}$ The expected products were extended from reverse primers (AR or BR) to the first forward primers. Each step extended over one primer bridged by the underlined overlapped sequences. As designed, the overlapped sequences in single-stranded primers next to each other were used as a bridge to convert the single-stranded primers into double-stranded products in which the underlined sequences were flanked by other sequences.

following steps, $1 \mu \mathrm{L}$ PCR product from the immediate previous reaction was added to the reaction system containing the relevant forward and reverse primers.

\subsection{Application of the De Novo Synthesized Templates in PCR Test}

Two pairs of primers targeting the de novo synthesized templates were used. The primer pairs of AF with the sequence of 5 'cgctctgataagagctacgagc 3 ' and AR (see Table 1) targeted Temp-I. And the primer pairs of BF with the sequence of 5'ctgcgcattgtctcc tggtttactg3' and BR (see Table 1) were used to target Temp-II. The de novo synthesized templates were gel purified with a DNA fragment purification kit (Takara, Dalian), and the concentrations were measured by ultraviolet (UV) spectrometry. The templates were then serially diluted by a fraction of 10 using nuclease-free $\mathrm{H}_{2} \mathrm{O}$. PCR was performed in a total volume of $25 \mu \mathrm{L}$ with the same conditions described in Subheading 2.2. after an initial denaturation of $2 \mathrm{~min}$ at $95^{\circ} \mathrm{C}$.

\section{Results}

As shown in Figure 1, DNA fragments in lengths of 182 and 204 bp were obtained from primers of set $\mathrm{A}$ and set $\mathrm{B}$, respectively, by the single-step sequential primer extension where each reaction contained four partially overlapping

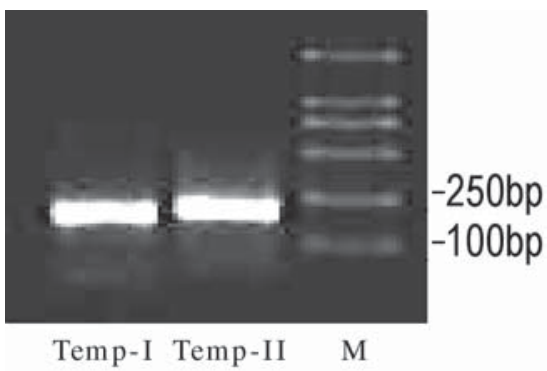

Fig. 1. A predominant single band was produced from the four primers of either primer set when the specified concentrations of the different primers were used. Temp-I was the product from primer set A and Temp-II was the product from primer set B. Intermediate products from steps 1 and 2 were difficult to detect because of the decreased amount of relevant primers used. $M$ represents DNA marker.

primers. The products of the single-step sequential primer extension were the same size as predicted in Table 2, equaling the summation of the four overlapping primers minus half of the overlapping nucleotides.

When consecutive steps of sequential primer extension were used, each step yielded a product equal to the size of the two partially overlapping primers minus half of the overlapping bases. The 
product of the next round reaction was always longer than the previous round. The longest desired products were those from the last steps, which were the final products of Temp-I and Temp-II (Fig. 2). The size of the final products was exactly the same whether they were produced from single-step or consecutive-step sequential primer extension. The consecutive-step sequential primer extension was used in case there were some primer sets in the single-step sequential primer extension that did not work. Sequence analysis confirmed that these two de novo synthesized templates were identical to the appropriate regions of SARS genome.

These two de novo synthesized templates were further evaluated in PCR reactions using different amounts of template. As shown in Figure 3, the templates obtained from the de novo synthesis worked well in testing primer pairs that targeted them. For reactions using 30 PCR cycles and 1000 or more copies of template, both the primer pair $\mathrm{AF}$ and $\mathrm{AR}$ and the primer pair BF and BR produced significant amount of PCR products. The amount of PCR product produced in these reactions correlated positively with the number of template copies used (Fig. 3).

\section{Discussion}

PCR reactions are widely used in both basic and clinical research, such as in the diagnosis of certain genetic and infectious diseases. The technological characteristics of PCR itself require the inclusion of both negative and positive controls, particularly when used in clinical applications. It is relatively simple in the diagnostic application in genetic diseases, whereas a positive control for the PCR kit used in infectious disease is more complicated and usually under stricter regulation. In the present study, we have reported a quick and safe strategy for de novo synthesis of DNA fragments complimentary to the SARS viral genome. The de novo synthesized DNA fragment can be used in the development of PCR diagnostic assays and in clinical PCR testing kits as a positive control. The de novo synthesis of known genes of interest can also be used in preparation for expres-

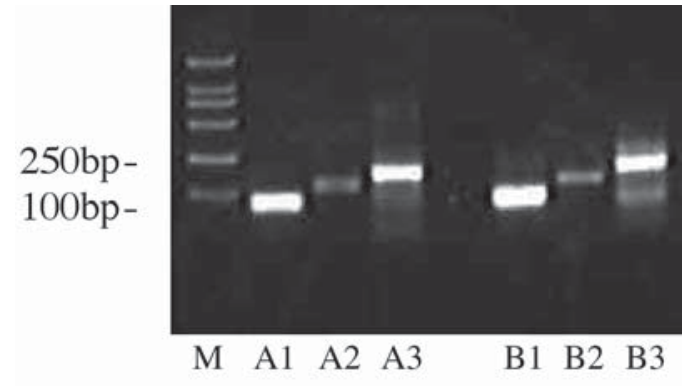

Fig. 2. An increasing size of products with increasing steps during consecutive-step sequential primer extension. $A 1, A 2$, and $A 3$ represent the products from steps 1, 2, and 3, respectively, for primer set A; $B 1$, $B 2$, and $B 3$ represent the products from steps 1,2 , and 3 , respectively, of the sequential primer extension for primer set B. $M$ represents DNA marker.

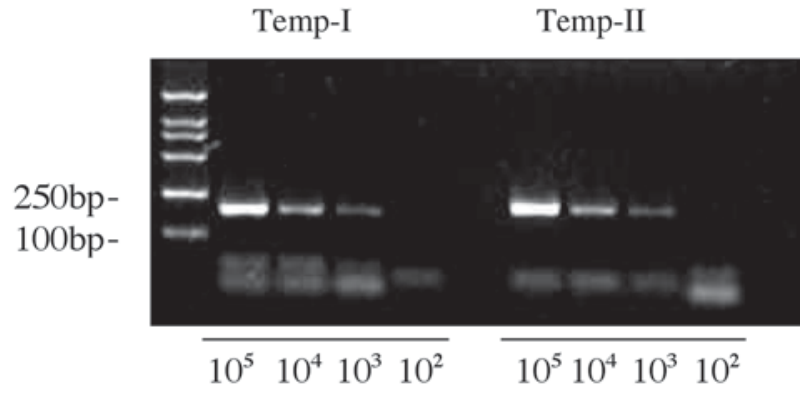

Copy number of templates employed

Fig. 3. Varying amount of PCR products amplified from different copy numbers of templates Temp-I and Temp-II. The amount of PCR products was significantly less when 100 copies of templates were used as compared with the products yielded from templates with 1000 or more copies. The two sets of primers showed similar sensitivities using the de novo synthesized PCR templates.

sion constructs for some specific proteins. In addition, the de novo synthesis of gene fragments using oligonucleotide sets is a straightforward strategy for mutagenesis studies.

Alternative methods for the production of templates are available but each has its own disadvantages. Chemical synthesis is rapid and routinely used for short oligonucleotides such as those shorter than 100 nucleotides. However, direct synthesis of long DNA fragment is technically lim- 


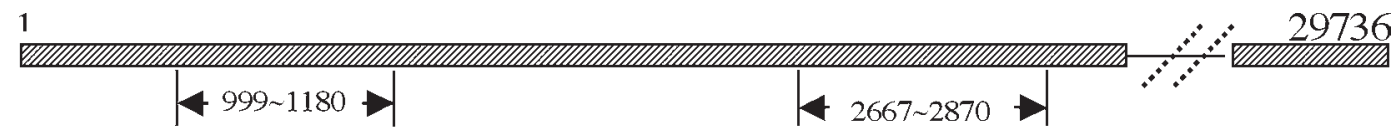

Temp I

$$
\begin{aligned}
& \text { tgataagagctacgagcaccagacacccttcgaaattaag } \\
& \text { agtgccaagaaatttgacactticaaaggggaatgcccaa } \\
& \text { agttgtgtttcctcttaactcaaaagtcaaagtcattcaacca } \\
& \text { cgtgttgaaaagaaaaagactgagggtttcatggggcgtat } \\
& \text { acgctctgtgtaccctg }
\end{aligned}
$$

Temp II

$$
\begin{aligned}
& \text { attgtctcctggtttactggctacaaacaatgicttcgcttaaa } \\
& \text { agggggtgcaccaattaaaggtgtaacctttggagaagata } \\
& \text { ctgttgggaagttcaaggttacaagaatgtgagaatcacatt } \\
& \text { tgagcttgatgaacgtgttgacaaagtgcttaatgaaaagtg } \\
& \text { ctctgtctacactgttgaatccggtaccgaagt }
\end{aligned}
$$

Fig. 4. Two gene fragments complementary to SARS virus TOR2 were obtained using the de novo gene synthesis technology. The Temp-I and Temp-II were complementary to 999-1180 and 2667-2870 of the SARS virus genome, respectively, as illustrated.

ited because the chemical reaction of each round is incomplete, thus the efficiency decreases with the propagation of reaction cycles. Taking advantage of chemical synthesis of oligonucleotides of fewer than 100 bases, the present strategy converted a set of partially overlapping, short, single-stranded oligonucleotides into a longer, double-stranded segment, simply by employing a new technology for the de novo synthesis of genes or gene fragments, sequential primer extension.

It is possible to chemically synthesize a set of double-stranded fragments with overhanging terminals compatible with each other and then to combine them by sequential ligation. Theoretically, both the sequential ligation and the sequential primer extension work for de novo synthesis of gene fragments. We did not test the sequential ligation strategy, as it is more expensive and time consuming. The urgent need in SARS diagnostic assay encouraged us to use sequential primer extension.

The outbreak of SARS demonstrates the need for rapid response to unknown diseases in the future. De novo synthesis of the PCR template complimentary to a viral genome provides a tool for the rapid development of early diagnostic assays for any new pathogen as soon as its sequence is known. For example, the two de novo synthesized DNA fragments described here can serve as positive controls in clinical PCR tests because theses two fragments are designed from the complimentary sequences of SARS coronavirus TOR2 (1) as shown in Figure 4. Although the sequential primer extension was developed for SARS diagnostic assay, the technology itself has wide applications in other fields in functional genomics. For instance, the de novo synthesis of a mutated, truncated, or chimeric gene encoding a specific protein will be possible and is very useful.

Taken together, the de novo synthesis of the PCR template reported in the present study provides a simple and quick technology in preparing a gene fragment of interest. The sequential primer extension is not technically difficult as compared to routine PCR, except for its requirement of a set of overlapping primers. The templates developed from this new method can be directly used as positive control in PCR assay. It is hoped that a wide application of the de novo synthesis of the PCR template will facilitate the development of a clinical PCR kit for the early diagnosis of SARS.

\section{Acknowledgments}

This research was partially supported from private financial sources provided by Mr. Yuebin Yang at Boda, Inc., and Xi-Kun Li and Yuexia Ban at Genomapping, Inc. The authors thank Xuemei Wu, Nan Jiang, and Lu Wang for their technical support. 


\section{References}

1. Marra, M. A, Jones, S. J, Astell, C. R., et al. (2003) The genome sequence of the SARS-associated coronavirus. Science 300, 1399-1404.

2. Drosten, C., Gunther, S., Preiser. W., et al. (2003) Identification of a novel coronavirus in patients with severe acute respiratory syndrome. N. Engl. J. Med. 348, 1967-1976.

3. Ksiazek, T. G., Erdman, D., Goldsmith, C. S., et al. (2003) A novel coronavirus associated with severe acute respiratory syndrome. N. Engl.J. Med. 348, 1953-1966.

4. World Health Organization. (2003) Use of laboratory methods for SARS diagnosis. Available at http:// www.who.int/csr/sars/labmethods/en/print.html. Accessed August 21, 2003.

5. Ashraf, H. (2003) Investigations continue as SARS claims more lives. Lancet 361, 1276.

6. Zhang, J., Li, K., Deng, Z., Liao, D., Fang, W., and Zhang, X. (2003) Efficient mutagenesis method for producing the template of single nucleotide polymorphisms. Mol. Biotechnol. 24, 105-110.

7. Horton, R. M., Cai, Z., Ho, S. N., and Pease, L. R. (1990) Gene splicing by overlap extension: tailormade genes using the polymerase chain reaction. Biotechniques 8, 528-535. 